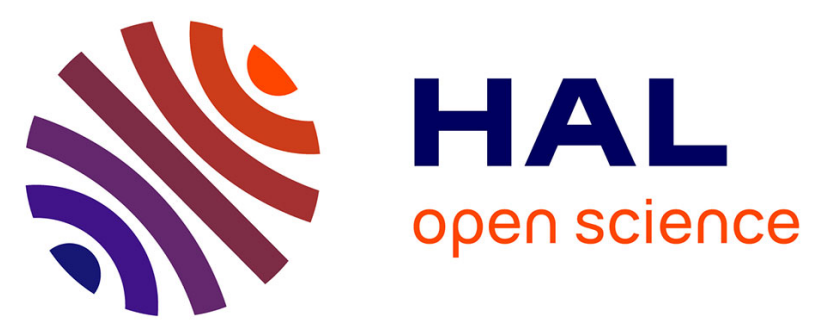

\title{
Joint hybrid ARQ and iterative space-time equalization for coded transmission over MIMO-ISI channel
}

\author{
Tarik Ait Idir, Houda Chafnaji, Samir Saoudi
}

\section{To cite this version:}

Tarik Ait Idir, Houda Chafnaji, Samir Saoudi. Joint hybrid ARQ and iterative space-time equalization for coded transmission over MIMO-ISI channel. WCNC 2008: IEEE wireless communications \& networking conference, March 31 - April 3, Las Vegas, USA, Mar 2008, Las Vegas, United States. pp.622 - 627, 10.1109/WCNC.2008.115 . hal-02120651

\section{HAL Id: hal-02120651 \\ https://hal.science/hal-02120651}

Submitted on 24 May 2019

HAL is a multi-disciplinary open access archive for the deposit and dissemination of scientific research documents, whether they are published or not. The documents may come from teaching and research institutions in France or abroad, or from public or private research centers.
L'archive ouverte pluridisciplinaire HAL, est destinée au dépôt et à la diffusion de documents scientifiques de niveau recherche, publiés ou non, émanant des établissements d'enseignement et de recherche français ou étrangers, des laboratoires publics ou privés. 


\title{
Joint Hybrid ARQ and Iterative Space-Time Equalization for Coded Transmission over the MIMO-ISI Channel
}

\author{
Tarik Ait-Idir ${ }^{(1,2)}$, Houda Chafnaji ${ }^{(1,2)}$, and Samir Saoudi ${ }^{(2)}$ \\ ${ }^{(1)}$ Communication Systems Department, INPT, Madinat Al Irfane, Rabat, Morocco \\ ${ }^{(2)}$ Signal and Communications Department, Telecom Bretagne, BP 832, 29285 Brest Cedex, France. \\ Emails: aitidir@ieee.org, \{houda.chafnaji,samir.saoudi\}@telecom-bretagne.eu
}

\begin{abstract}
This paper focuses on the problem of efficient packet combining techniques for coded systems with hybrid automatic repeat request (ARQ) protocols operating over the frequency selective fading multiple input multiple output (MIMO) channel. We introduce a receiver scheme where space-time soft equalization is integrated into the packet combiner. This allows to create at each transmission an additional set of virtual receive antennas, thereby increasing the diversity order of the system. We also propose a second packet combining scheme where equalization is performed separately for each transmission. Iterative channel estimation is also investigated, and a training scheme where the pilot symbols are not retransmitted is introduced. Block error rate (BLER) performance is investigated via computer simulations for both perfect and imprecise channel state information (CSI) at the receiver side for demonstrating the potential of integrated soft equalization-based packet combining for transmission over multipath MIMO channels.
\end{abstract}

\section{INTRODUCTION}

Hybrid automatic repeat request (ARQ) protocols and multiple input multiple output (MIMO) space-time multiplexing oriented architectures are two major techniques for the evolution of wireless high speed packet access networks [1]. While MIMO multiplexing techniques allow to increase the spectrum efficiency through the transmission of different parallel substreams over multiple antennas [2], hybrid ARQ protocols perform packet combining of different packet transmissions to increase the system throughput. In the literature, several packet combining strategies have been proposed. In [3], Chase has developed a maximum likelihood (ML)-based technique for combining multiple coded packets. In [4], the authors have introduced different diversity-based combining schemes for hybrid ARQ systems.

Recently, particular attention has been paid to the design of packet combining algorithms for MIMO systems. In general, multi-antenna transmission techniques provide multiplexing and diversity gains. If a ARQ protocol is employed at the upper layer, retransmission of the same data packet increases the diversity gain, especially if the MIMO channel changes from one transmission to another. In addition, the presence of intersymbol interference (ISI) caused by frequency selective fading translates into additional diversity branches if an efficient equalization scheme is employed. Therefore, the maximum achievable diversity order of a multi-antenna transmission link with ARQ over a time varying MIMOISI fading channel is $k L N_{R}$, where $k, L$, and $N_{R}$ are the numbers of transmissions, MIMO paths, and receive antennas, respectively. Symbol-level combining techniques based on zero forcing (ZF) and minimum mean square error (MMSE) filtering have been investigated for MIMO systems with flat fading [5]. MIMO ARQ techniques with diversity mapping and sphere decoding-based joint detection and packet combining has been proposed for a flat fading multi-antenna channel [6]. In [7], [8], performance of turbo coded hybrid ARQ with iterative MMSE frequency domain equalization (FDE) for single carrier transmission over the broadband MIMO channel has been evaluated.

In this paper, we focus on efficient packet combining techniques for ARQ with space-time bit interleaved coded modulation (STBICM) systems operating over a MIMOISI channel. Several contributions have confirmed the remarkable diversity gain of STBICM with iterative decoding over broadband multi-antenna fading channels [9], [10], [11]. Efficient low-complexity turbo equalization strategies for STBICM using soft interference cancellation (IC) techniques and MMSE filtering have been proposed in the last few years [12], [13], [14]. Cyclic prefix (CP)-aided iterative equalization approaches relying on frequency domain processing have recently shown an attractive performance to complexity tradeoff [15], [16]. In the framework of an STBICM transmission with ARQ and iterative decoding, the packet combining scheme has a great impact on the achievable diversity order and consequently the overall receiver performance. This paper introduces a new combining technique for ARQ MIMOISI systems where space-time equalization is integrated into the packet combiner. The idea of merging soft equalization and transmission combining has been initially introduced by Samra and Ding for single antenna systems using a maximum a posteriori (MAP) equalizer [17]. The authors have shown, for a coded transmission scenario, that packet combining with integrated equalization outperforms iterative combining techniques [18] where the ARQ protocol applies different interleavers for different transmissions, and the receiver iterates over multiple equalizers before channel decoding. ARQ with integrated equalization has been then applied to MIMO channels with flat fading using a sphere decoding-based joint detection scheme [6]. The key idea of our receiver is to view a particular transmission $k$ as an additional set of virtual $N_{R}$ receive antennas. Low-complexity time domain space-time soft IC and MMSE equalization is then performed to exploit the $k L N_{R}$ available diversity branches. We also introduce a combining scheme quite similar to the iterative combining concept of [18], and show that the integrated equalization 
approach gives better performance than the one with separate equalization stages. We also focus on the performance of the proposed technique when imprecise channel state information (CSI) is available at the receiver side, and introduce a training scheme where the pilot symbols are transmitted only during the first transmission.

The remainder of the paper is organized as follows. In Section II, we introduce the hybrid ARQ communication model. Section III details the proposed combining schemes with turbo equalization then focuses on complexity issues. Numerical results are provided in Section IV. The paper is concluded is Section V.

\section{HybRid ARQ COMMUNICATION MODEL}

We consider a point to point multi-antenna system operating over a frequency selective fading channel and employing an ARQ scheme at the upper layer. Each data block is initially transmitted. Upon the reception of a negative acknowledgment (NACK) message, subsequent retransmissions occur until the packet is correctly received or a preset maximum number of retransmissions $K$ is reached. Transmission of an information block includes channel coding using a rate- $\rho$ encoder, interleaving with the aid of a semi-random interleaver $\Pi$, then bit to symbol mapping, and spatial multiplexing over $N_{T}$ transmit antennas. This presents a typical STBICM scheme. The constellation set is denoted $\mathcal{S}$. The mapping function relating each group of coded and interleaved bits $b_{1, t, i}, \cdots, b_{M, t, i}$ to symbol $s_{t, i}$ is denoted $\varphi:\{0,1\}^{M} \rightarrow$ $\mathcal{S}$, where $M=\log _{2}|\mathcal{S}|$, and $t=1, \cdots, N_{T}$, and $i=$ $0, \cdots, T-1$ denote transmit antenna, and channel use indices, respectively. Transmission occurs on a block fading quasistatic MIMO-ISI channel comprised of $L$ discrete MIMO taps. The number of paths is supposed to be constant at least for $K$ consecutive transmissions. The $N_{R} \times N_{T}$ channel matrices realizations during retransmission number $k=1, \cdots, K$, and corresponding to paths $l=0, \cdots, L-1$ are denoted $\mathbf{H}_{0}^{(k)}, \cdots, \mathbf{H}_{L-1}^{(k)}$, respectively. Their coefficients are zeromean circularly symmetric complex Gaussian random variables verifying the following channel energy normalization condition,

$$
\mathbb{E}\left[\operatorname{diag}\left\{\sum_{l=0}^{L-1} \mathbf{H}_{l}^{(k)} \mathbf{H}_{l}^{(k)^{H}}\right\}\right]=N_{T} \mathbf{I}_{N_{R}} .
$$

We suppose that no knowledge of the CSI is available at the transmitter side, and perfect channel estimation is performed in reception. Equal power transmission strategy among the $N_{T}$ transmit antennas is therefore assumed,

$$
\mathbb{E}\left[\mathbf{s}_{i} \mathbf{s}_{i}^{H}\right]=\mathbf{I}_{N_{T}},
$$

where $\mathbf{s}_{i}=\left[s_{1, i}, \cdots, s_{N_{T}, i}\right]^{T} \in \mathcal{S}^{N_{T}}$ is the vector of transmitted symbols at channel use $i$. Note that the retransmitted packets go through the same STBICM transmitter. Puncturing and diversity mapping optimization are therefore out of the scope of this paper. Thus, at the $k$ th transmission, the $N_{R} \times 1$ received signal vector at discrete time $i$ is expressed as,

$$
\mathbf{y}_{i}^{(k)}=\sum_{l=0}^{L-1} \mathbf{H}_{l}^{(k)} \mathbf{s}_{i-l}+\mathbf{n}_{i}^{(k)}
$$

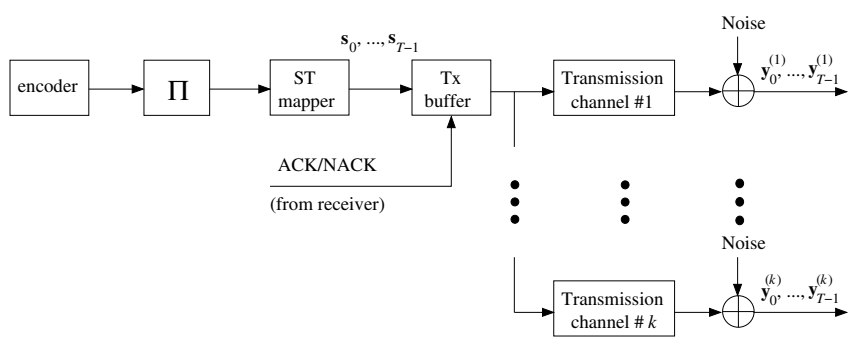

Fig. 1. STBICM transmitter with ARQ over a time-varying MIMO-ISI communication channel

where $\mathbf{n}_{i}^{(k)} \sim \mathcal{N}\left(\mathbf{0}_{N_{R} \times 1}, \sigma^{2} \mathbf{I}_{N_{R}}\right)$ is the thermal noise at the receiver side. Fig. 1 depicts the considered STBICM scheme with multiple retransmissions over different multiantenna channels.

\section{JOINT Hybrid ARQ AND SPACE-TIME SofT EQUALIZATION}

In this section, we introduce two efficient schemes for performing ARQ jointly with soft interference cancellation and MMSE-based equalization for transmission over MIMOISI channels. Then, we emphasis on their respective computational complexities.

\section{A. Hybrid ARQ with Integrated MMSE-Based Space-Time Equalization}

Transmission of the same symbol vectors $\mathbf{s}_{0}, \cdots, \mathbf{s}_{T-1}$ over different MIMO-ISI channels induces additional spatial and temporal diversities. After $k$ transmissions, the natural way for capturing all the diversity branches available in received signals $\mathbf{y}_{0}^{(1)}, \cdots, \mathbf{y}_{T-1}^{(k)}$ is to perform combination of transmitted blocks at the signal level. Thus, we consider retransmissions as additional sets of virtual receive antennas. Therefore, the system can be viewed, at the $k$ th transmission, as a point to point MIMO link with $N_{T}$ transmit and $k N_{R}$ receive antennas. Efficient equalization techniques relying on soft interference cancellation and iterative processing can be adopted to achieve the diversity order of $k L N_{R}$ provided by such a system. The receiver buffers received signals corresponding to all transmissions to obtain the $k N_{R} \times 1$ virtual received signal at channel use $i$ as

$$
\underline{\mathbf{y}}_{i}^{(k)} \triangleq\left[\mathbf{y}_{i}^{(1)^{T}}, \cdots, \mathbf{y}_{i}^{(k)^{T}}\right]^{T}
$$

which can be written according to the following $k N_{R} \times N_{T}$ MIMO-ISI communication model,

$$
\underline{\mathbf{y}}_{i}^{(k)}=\sum_{l=0}^{L-1} \underline{\mathbf{H}}_{l}^{(k)} \mathbf{s}_{i-l}+\underline{\mathbf{n}}_{i}^{(k)},
$$

where $\underline{\mathbf{n}}_{i}^{(k)}$ is a $k N_{R} \times 1$ zero-mean Gaussian noise vector with covariance $\sigma^{2} \mathbf{I}_{k N_{R}}$ and is given by

$$
\underline{\mathbf{n}}_{i}^{(k)} \triangleq\left[\mathbf{n}_{i}^{(1)^{T}}, \cdots, \mathbf{n}_{i}^{(k)^{T}}\right]^{T}
$$

and matrix $\underline{\mathbf{H}}_{l}^{(k)} \in \mathbb{C}^{k N_{R} \times N_{T}}$ groups channel matrices of the $l$ th tap until the $k$ th transmission,

$$
\underline{\mathbf{H}}_{l}^{(k)} \triangleq\left[\begin{array}{c}
\mathbf{H}_{l}^{(1)} \\
\vdots \\
\mathbf{H}_{l}^{(k)}
\end{array}\right] .
$$




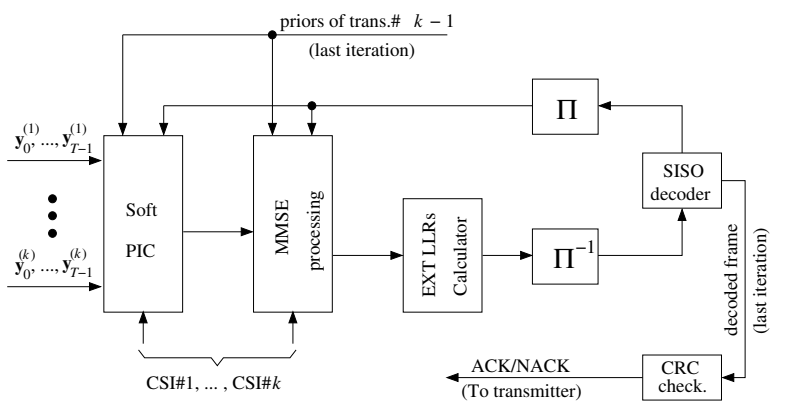

Fig. 2. Iterative STBICM receiver at the $k$ th transmission with ARQ and integrated MMSE turbo equalization

For performing ISI cancellation, let us consider the length- $L_{F}$ sliding-window detector whose output at discrete time $i$ is,

$$
\underline{\mathbf{y}}_{i_{L_{F}}}^{(k)} \triangleq\left[\underline{\mathbf{y}}_{i+L_{1}}^{(k)^{T}}, \cdots, \underline{\mathbf{y}}_{i-L_{2}}^{(k)^{T}}\right]^{T} \in \mathbb{C}^{k N_{R} L_{F}},
$$

where $L_{1}$ and $L_{2}$ are the forward and backward filter lengths, and $L_{F}=L_{1}+L_{2}+1$. With respect to (5), the sliding-window output $\underline{\mathbf{y}}_{i_{L_{F}}}^{(k)}$ can be written according to the following signal model,

$$
\underline{\mathbf{y}}_{i_{L_{F}}}^{(k)}=\underline{\mathbf{H}}^{(k)} \mathbf{s}_{i_{L_{F}}}+\underline{\mathbf{n}}_{i_{L_{F}}}^{(k)},
$$

where

$$
\begin{gathered}
\mathbf{s}_{i_{L_{F}}} \triangleq\left[\mathbf{s}_{i+L_{1}}^{T}, \cdots, \mathbf{s}_{i-L_{2}-L+1}^{T}\right]^{T} \in \mathcal{S}^{\left(L_{F}+L-1\right) N_{T}} \\
\underline{\mathbf{n}}_{i_{L_{F}}}^{(k)} \triangleq\left[\underline{\mathbf{n}}_{i+L_{1}}^{(k)^{T}}, \cdots, \underline{\mathbf{n}}_{i-L_{2}}^{(k) T^{T}}\right]^{T} \in \mathbb{C}^{k N_{R} L_{F}}
\end{gathered}
$$

and $\underline{\mathbf{H}}^{(k)} \in \mathbb{C}^{k N_{R} L_{F} \times\left(L_{F}+L-1\right) N_{T}}$ is a block Toeplitz matrix defined as,

$$
\underline{\mathbf{H}}^{(k)} \triangleq\left[\begin{array}{ccccc}
\underline{\mathbf{H}}_{0}^{(k)} & \cdots & \underline{\mathbf{H}}_{L-1}^{(k)} & & \mathbf{0} \\
& \ddots & & \ddots & \\
\mathbf{0} & & \underline{\mathbf{H}}_{0}^{(k)} & \cdots & \underline{\mathbf{H}}_{L-1}^{(k)}
\end{array}\right] .
$$

At the $k$ th transmission, the receiver makes use of loglikelihood ratio (LLR) values computed during the last iteration of the previous transmission $k-1$, and the communication model (9) for performing soft space-time equalization and computing extrinsic LLRs about coded and interleaved bits. The equalization and decoding processes are then iteratively conducted through the exchange of extrinsic information.

Let $\phi_{t, i}=\left[\phi_{1, t, i}, \cdots, \phi_{M, t, i}\right]^{T} \in \mathbb{R}^{M}$ denotes the vector of a priori LLRs corresponding to coded and interleaved bits $b_{1, t, i}, \cdots, b_{M, t, i}$ generated by the soft decoder. For the sake of notation simplicity, the transmission index $k$ is dropped form the LLR values. The hybrid ARQ combiner with integrated space-time equalization cancels soft ISI for each symbol $s_{t, i}$ in a parallel interference cancellation (PIC) fashion, with the aid of the sliding-window output $\underline{\mathbf{y}}_{i_{L_{F}}}^{(k)}$ at transmission number $k$ and the soft estimates of interfering symbol vectors. The $k N_{R} L_{F} \times 1$ "ISI free" signal vector corresponding to the detection of symbol $s_{t, i}$ is obtained as,

$$
\underline{\mathbf{z}}_{t, i}^{(k)} \triangleq \underline{\mathbf{y}}_{i_{L_{F}}}^{(k)}-\underline{\mathbf{H}}^{(k)} \tilde{\mathbf{s}}_{i_{L_{F}} \mid t},
$$

where the soft interference vector $\tilde{\mathbf{s}}_{i_{L_{F}} \mid t}$ is computed according to the following conditional mean,

$$
\tilde{\mathbf{s}}_{i_{L_{F}} \mid t} \triangleq \mathbb{E}\left[\mathbf{s}_{i_{L_{F}}} \mid \phi_{t^{\prime}, i^{\prime}}:\left(t^{\prime}, i^{\prime}\right) \neq(t, i)\right] .
$$

MMSE processing is then performed to produce a decision statistic $z_{t, i}^{(k)}$ serving for the computation of extrinsic LLRs. We use unconditional MMSE filtering as it is an efficient method for performing turbo MMSE equalization with lowcomplexity [12], [13]. The output of the MMSE filter is,

$$
z_{t, i}^{(k)} \triangleq \mathbf{e}_{t}^{H} \underline{\mathbf{H}}^{(k)^{H}}\left(\underline{\mathbf{H}}^{(k)} \boldsymbol{\Theta}_{t} \underline{\mathbf{H}}^{(k)^{H}}+\sigma^{2} \mathbf{I}_{k N_{R} L_{F}}\right)^{-1} \underline{\mathbf{z}}_{t, i}^{(k)},
$$

where $\mathbf{e}_{t}$ denotes the $\left(L_{1} N_{T}+t\right)$ th vector of the canonical basis, and $\Theta_{t}$ is an unconditional covariance matrix approximated by the time average of conditional covariance $\boldsymbol{\Theta}_{t, i}$ defined as,

$$
\begin{aligned}
\boldsymbol{\Theta}_{t, i} \triangleq \mathbb{E}\left[\left(\mathbf{s}_{i_{L_{F}}}-\tilde{\mathbf{s}}_{i_{L_{F}}}\right)\right. & \left(\mathbf{s}_{i_{L_{F}}}-\tilde{\mathbf{s}}_{i_{L_{F}}}\right)^{H} \\
& \left.\mid \phi_{t^{\prime}, i^{\prime}}:\left(t^{\prime}, i^{\prime}\right) \neq(t, i)\right],
\end{aligned}
$$

where $\tilde{\mathbf{s}}_{i_{L_{F}}}$ is the conditional mean of $\mathbf{s}_{i_{L_{F}}}$. The extrinsic LLR value $\phi_{m, t, i}^{(e)}$ corresponding to coded and interleaved bit $b_{m, t, i}$ is then computed as,

$\phi_{m, t, i}^{(e)}=\log \frac{\sum_{s \in \mathcal{S}_{m, 1}} p\left(z_{t, i}^{(k)} \mid s\right) \cdot \exp \left\{\sum_{m^{\prime} \neq m} \varphi_{m^{\prime}}^{-1}(s) \phi_{m^{\prime}, t, i}\right\}}{\sum_{s \in \mathcal{S}_{m, 0}} p\left(z_{t, i}^{(k)} \mid s\right) \cdot \exp \left\{\sum_{m^{\prime} \neq m} \varphi_{m^{\prime}}^{-1}(s) \phi_{m^{\prime}, t, i}\right\}}$,

where

$$
\begin{aligned}
& \mathcal{S}_{m, 1}=\left\{s \in \mathcal{S} \mid \varphi_{m}^{-1}(s)=1\right\} \\
& \mathcal{S}_{m, 0}=\left\{s \in \mathcal{S} \mid \varphi_{m}^{-1}(s)=0\right\} .
\end{aligned}
$$

The block diagram of the iterative receiver with integrated equalization is depicted in Fig. 2.

Note that during the first turbo iteration, the iterative ARQ receiver with integrated equalization, makes use of a priori LLRs of coded and interleaved bits generated by the soft input soft output (SISO) decoder during the last iteration of the previous transmission $k-1$. Therefore, interference cancellation is performed from the first iteration. This idea was initially introduced by [19] in the context of single antenna coded systems with ARQ.

\section{B. Hybrid ARQ and MMSE-Based Space-Time Equalization with Filter Outputs Combining}

In this subsection, we present a second receiver scheme for combining multiple transmissions. Instead of performing packet combining at the signal level and therefore increasing the dimensionality of the problem through additional virtual receive antennas, we perform space-time equalization separately for each transmission then combine the outputs of the MMSE filters corresponding to multiple transmissions for computing extrinsic LLRs. The block diagram of the proposed receiver is presented in Fig. 3.

At the $k$ th transmission, MMSE-based space-time equalization is performed using only signals $\mathbf{y}_{0}^{(k)}, \cdots, \mathbf{y}_{T-1}^{(k)}$ received 


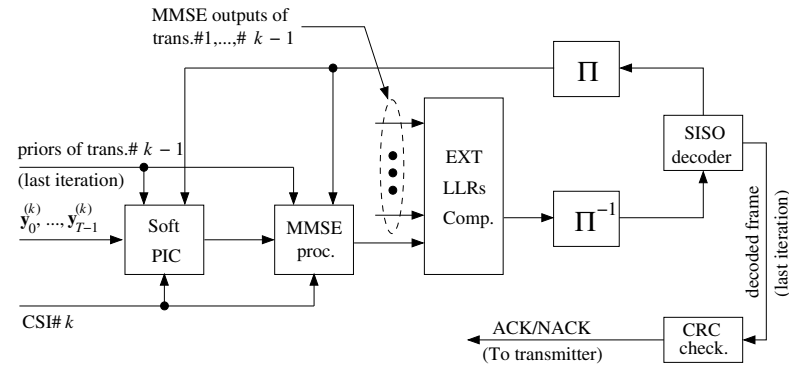

Fig. 3. Iterative STBICM receiver at the $k$ th transmission with ARQ and filter outputs combining

at transmission $k$. The sliding-window communication model is,

$$
\mathbf{y}_{i_{L_{F}}}^{(k)}=\mathbf{H}^{(k)} \mathbf{s}_{i_{L_{F}}}+\mathbf{n}_{i_{L_{F}}}^{(k)}
$$

where

$$
\begin{aligned}
& \mathbf{y}_{i_{L_{F}}}^{(k)} \triangleq\left[\mathbf{y}_{i+L_{1}}^{(k)^{T}}, \cdots, \mathbf{y}_{i-L_{2}}^{(k)^{T}}\right]^{T} \in \mathbb{C}^{N_{R} L_{F}}, \\
& \mathbf{n}_{i_{L_{F}}}^{(k)} \triangleq\left[\mathbf{n}_{i+L_{1}}^{(k)^{T}}, \cdots, \mathbf{n}_{i-L_{2}}^{(k)^{T}}\right]^{T} \in \mathbb{C}^{N_{R} L_{F}},
\end{aligned}
$$

and $\mathbf{H}^{(k)} \in \mathbb{C}^{N_{R} L_{F} \times\left(L_{F}+L-1\right) N_{T}}$ is the block Toeplitz matrix corresponding to transmission $k$, and is defined as,

$$
\mathbf{H}^{(k)} \triangleq\left[\begin{array}{ccccc}
\mathbf{H}_{0}^{(k)} & \cdots & \mathbf{H}_{L-1}^{(k)} & & \mathbf{0} \\
& \ddots & & \ddots & \\
\mathbf{0} & & \mathbf{H}_{0}^{(k)} & \cdots & \mathbf{H}_{L-1}^{(k)}
\end{array}\right]
$$

Soft ISI cancellation and unconditional MMSE processing are performed similarly to (13) and (15), respectively. Let $x_{t, i}^{(k)}$ denotes the complex scalar output of the $k$ th transmission's MMSE filter corresponding to antenna $t$. The ARQ receiver with filter outputs combining computes at the $k$ th transmission the extrinsic LLR value $\phi_{m, t, i}^{(e)}$ corresponding to coded and interleaved $b_{m, t, i}$ with the aid of the decision vector $\mathbf{x}_{t, i}^{(k)} \triangleq$ $\left[x_{t, i}^{(1)}, \cdots, x_{t, i}^{(k)}\right]^{T} \in \mathbb{C}^{k}$ as,

$\phi_{m, t, i}^{(e)}=\log \frac{\sum_{s \in \mathcal{S}_{m, 1}} p\left(\mathbf{x}_{t, i}^{(k)} \mid s\right) \cdot \exp \left\{\sum_{m^{\prime} \neq m} \varphi_{m^{\prime}}^{-1}(s) \phi_{m^{\prime}, t, i}\right\}}{\sum_{s \in \mathcal{S}_{m, 0}} p\left(\mathbf{x}_{t, i}^{(k)} \mid s\right) \cdot \exp \left\{\sum_{m^{\prime} \neq m} \varphi_{m^{\prime}}^{-1}(s) \phi_{m^{\prime}, t, i}\right\}}$,

where subsets $\mathcal{S}_{m, 1}$ and $\mathcal{S}_{m, 0}$ are those defined in (18). Note that $x_{t, i}^{(1)}, \cdots, x_{t, i}^{(k-1)}$ represent the $t$ th antenna MMSE filter outputs corresponding to the last iteration of transmissions $1, \cdots, k-1$, respectively.

\section{Computational Complexity Issues}

Packet combining with integrated MMSE-based spacetime equalization is mainly limited by the computation of $N_{T}$ MMSE filters (15) needed for ISI equalization. This involves the inversion of $N_{T}$ matrices of size $k N_{R} L_{F} \times$ $k N_{R} L_{F}$ at the $k$ th transmission. Therefore, this combining algorithm has a complexity equal to $O\left(k^{3} N_{R}^{3} L_{F}^{3}\right)$ for each antenna. Extrinsic information computation is performed with respect to (17) which is exponential in $|\mathcal{S}|$. The MMSE filter outputs-based packet combining algorithm performs spacetime soft equalization separately for each transmission then combines the MMSE filter outputs of the current and the previous transmissions according to (23). This presents a complexity equal to $O\left(N_{R}^{3} L_{F}^{3}\right)$ for each antenna at each transmission. In addition, assuming independence between MMSE filter outputs $x_{t, i}^{(1)}, \cdots, x_{t, i}^{(k)}$ of different transmissions, the covariance matrix of residual ISI plus thermal noise in the $k \times 1$ decision vector $\mathbf{x}_{t, i}^{(k)}$ is diagonal. Therefore, the metric in (23) serving for extrinsic LLRs computation has a complexity equal to $O\left(2^{|\mathcal{S}|}\right)$. Transmission combining using integrated equalization is consequently cubic in $k$, while the filter outputs-based combining approach is only linear in the number of transmissions. This complexity impairment is motivated in the simulation section by the dramatic diversity order the packet combining with integrated equalization-based approach can achieve. Note that in contrast to [18], the filter outputs combining-based technique avoids iterating over different equalizers.

\section{PERFormance Evaluation}

\section{A. Simulation Settings}

Simulations are conducted for an STBICM transmission link with $N_{R}=2$ receive antennas, quadrature phase shift keying (QPSK) modulation, four states convolutional encoder with polynomial generators $(5,7)_{8}$. The length of the coded block is 1032 bits including tails. The channel is supposed to change independently from one transmission to another. Two MIMO-ISI channel profiles with two and four EQual (EQ) power taps are considered to evaluate the performance of the proposed algorithms for different frequency selectivity scenarios. The SISO decoder employs the Max-Log-MAP version of the MAP decoding algorithm [20]. The iterative packet combining, equalization and decoding process is stopped after five turbo iterations. The total number of transmissions is set to $K=3$. Performance is evaluated in terms of block error rate (BLER) versus $E_{b} / N_{0}$ per transmission, where $E_{b}$ denotes the average energy per information bit per receive antenna. We focus on packet error rate per transmission because improving the BLER performance implies reducing the number of transmissions and therefore increasing the throughput. For each transmission $k=1,2$, and 3 , we employ the matched filter bound (MFB) for evaluating the diversity order achieved by the proposed algorithms. The MFB corresponds to perfect ISI cancellation and maximal ratio combining (MRC) of the $k L N_{R}$ diversity branches available at the receiver side at transmission $k$. In the following, ARQ with integrated equalization and MMSE filters outputs combining schemes are labeled Receiver1 and Receiver2, respectively.

\section{B. Performance Evaluation with Perfect CSI}

We start by evaluating the BLER performance of the considered STBICM system with $N_{T}=2$ transmit antennas. This corresponds to a spectrum efficiency of two information bits per channel use (2b/c.u). First, we consider a 2EQ MIMO-ISI channel profile. The sliding-window length is set to $L_{F}=2\left(L_{1}=1, L_{2}=0\right)$. Fig. 4 plots the BLER performance of Receiver1 and Receiver2. We observe that 


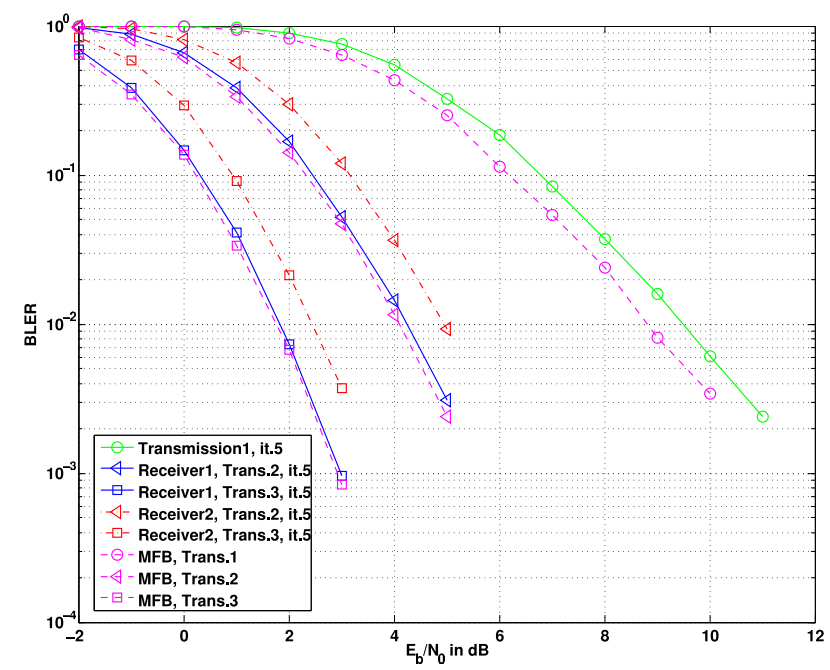

Fig. 4. BLER performance of the proposed receivers for an STBICM with $N_{T}=N_{R}=2$ and $2 \mathrm{EQ}$ channel profile

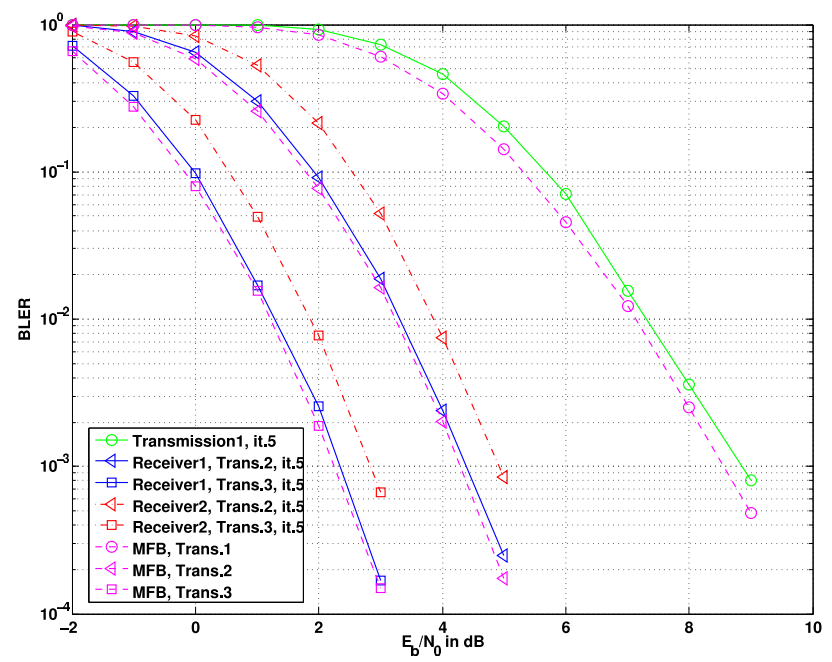

Fig. 5. BLER performance of the proposed receivers for an STBICM with $N_{T}=N_{R}=2$ and $4 \mathrm{EQ}$ channel profile

additional transmissions induce a dramatic performance improvement. This is due to the increase of the diversity order, i.e. four in the first transmission, and eight and twelve in the second and the third transmissions, respectively. The gap between ARQ with integrated equalization and filter outputs combining is about $0.67 \mathrm{~dB}$ at $10^{-2}$ BLER for both the second and the third transmissions. The MFB performance is almost achieved by Receiver1. Second, we examine the BLER performance for a $4 \mathrm{EQ}$ channel profile. The filter length is $L_{F}=17\left(L_{1}=8, L_{2}=8\right)$. The obtained performance is presented in Fig. 5. As noted in the case of $2 \mathrm{EQ}, \mathrm{ARQ}$ with integrated equalization achieves the maximum diversity order at each transmission. The ISI effect is perfectly turned into a diversity gain which is equal to $0.66 \mathrm{~dB}$ at $10^{-3} \mathrm{BLER}$ compared with a $2 \mathrm{EQ}$ channel profile. We now turn to the case of overloaded configurations (i.e. $N_{T}>N_{R}$ ) and consider an STBICM with $N_{T}=4$ transmit antennas. This corresponds to a spectrum efficiency of $4 \mathrm{~b} / \mathrm{c} . u$. The MIMO-ISI channel has 2EQ taps, and the sliding-window length is set to $L_{F}=13$ $\left(L_{1}=6, L_{2}=6\right.$ ). Fig. 6 plots the obtained performance for

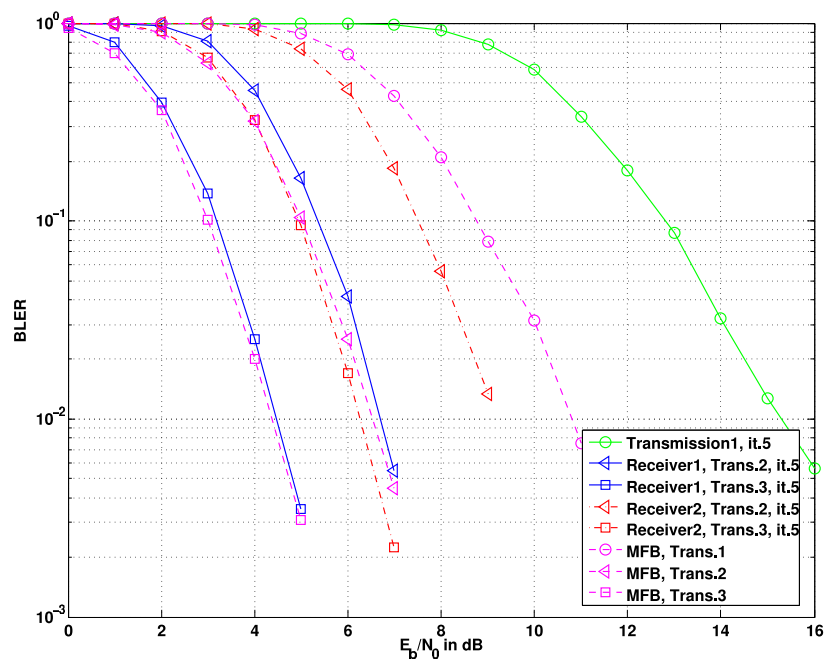

Fig. 6. BLER performance of the proposed receivers for an STBICM with $N_{T}=4, N_{R}=2$, and 2EQ channel profile

three transmissions. ARQ with integrated equalization clearly outperforms ARQ with filter outputs combining. While Receiver1 mostly achieves the MFB in both the second and the third transmissions, Receiver2 still around $2 \mathrm{~dB}$ far from the MFB. This performance gap is mainly due to the combining strategy in ARQ with integrated equalization where the number of "receive" antennas is increased in each transmission, which relaxes the overloading constraint.

\section{Performance Evaluation with Iterative CSI Estimation}

In this subsection, we focus on the performance of Receiver1 when imprecise CSI is present in reception. In the case of a MIMO-ISI channel with severe frequency selectivity, channel estimation becomes a complicated task because of the huge number of channel coefficients to be estimated. This translates into a large length of the pilot sequence and, therefore, a dramatic reduction in the system throughput. When iterative processing is adopted at the receiver side, an alternative solution to this problem consists on introducing the channel estimator in the turbo loop. At each iteration, additional pilots are constructed with the aid of the a priori information delivered by the SISO decoder, which allows to refine the channel estimate at each iteration. Several iterative channel estimation techniques have been proposed for MIMO systems. In this paper we consider an MMSE-based soft input channel estimator for MIMO-ISI channels similar to the one introduced in [21], [14]. The STBICM scheme has $N_{T}=2$ transmit antennas and operates over a 2EQ channel. A $2 \times 24$ pilot symbol matrix is appended to each transmitted block for performing CSI estimation during the first turbo iteration. Note that starting from the second transmission, the soft information produced by the SISO decoder during the last iteration of the previous transmission can be used for performing CSI estimation in the current transmission. Therefore, we consider two training schemes:

Scheme1: Corresponds to the case where pilots are appended to the data block at each transmission $k=1,2$, and 3. CSI estimation is performed at each transmission using both the training symbols and the soft information. The loss in $E_{b} / N_{0}$ 


\begin{tabular}{|c|c|c|c|c|c|c|}
\hline \multirow[b]{2}{*}{ Scheme 1} & \multicolumn{2}{|c|}{ Trans \#1 } & \multicolumn{2}{|c|}{ Trans \#2 } & \multicolumn{2}{|c|}{ Trans \#3 } \\
\hline & Pilots & Data & Pilots & Data & Pilots & Data \\
\hline & \multicolumn{2}{|c|}{ Trans \#1 } & \multicolumn{2}{|c|}{ Trans \#2 } & \multicolumn{2}{|c|}{ Trans \#3 } \\
\hline Scheme 2 & Pilots & Data & & Data & & Data \\
\hline
\end{tabular}

Fig. 7. Training with (Scheme1) and without (Scheme2) pilots in the subsequent transmissions

due to pilots insertion is about $0.39 \mathrm{~dB}$ (see Fig. 7).

Scheme2: Pilots are sent only in the first transmission. In the subsequent transmissions, the CSI is estimated only with the aid of the soft information. In this scheme, the loss in $E_{b} / N_{0}$ is reduced to $0.13 \mathrm{~dB}$ (see Fig. 7).

The BLER performance of the two considered schemes is presented in Fig. 8. We observe that surprisingly, Scheme2 outperforms Scheme1 by approximately $0.34 \mathrm{~dB}$ in both the second and the third transmissions. This is due to the fact that the performance gain offered by a training retransmission strategy is less than the pilot sequence insertion loss. This performance gap might be larger in the case of MIMO-ISI channels with severe frequency selectivity where longer pilots are needed.

\section{Conclusions}

In this paper, we have introduced two receiver schemes for performing packet combining jointly with space-time turbo equalization for coded MIMO-ISI systems. The first algorithm integrates equalization into the packet combiner by considering a transmission as an additional set of virtual receive antennas. The second one performs turbo equalization separately for each transmission then combines filter outputs. Simulations have demonstrated a superior performance for the first scheme compared with the second one, especially for overloaded systems. Iterative channel estimation has been also investigated, and we have shown that in the case of packet combining with integrated equalization, the pilot symbols can be sent only during the first transmission, and the CSI estimation might rely only on the soft information in the subsequent transmissions.

\section{REFERENCES}

[1] J. Peisa, S. Wager, M. Sagfors, J. Torsner, B. Goransson, T. Fulghum, C. Cozzo, and S. Grant, "High speed packet access evolution - concept and technologies," 65th IEEE veh. tech. conf. VTC'07 Spring, Dublin, Ireland, Apr. 2007.

[2] P. W. Wolniansky, G. J. Foschini, and G. D. Valenzuela, "V-BLAST : an architecture for realizing very high data rates over the rich scattering wireless channel," in Proc. Int. Symp. Signals, Systems, Electron. , Pisa, Italy, Sep. 1998.

[3] D. Chase, "Code combining-a maximum-likelihood decoding approach for combining an arbitrary number of noisy packets," IEEE Trans. Commun., vol. COM-33, no. 5, pp. 385-393, May 1985.

[4] B. Harvey, and S. Wicker, "Packet combining systems based on the Viterbi decoder," IEEE Trans. Commun., vol. 42, no. 2-4, pp. 1544 1557, Feb.-Apr. 1994

[5] E. N. Onggosanusi, A. G. Dabak, Y. Hui, and G. Jeong, "Hybrid ARQ transmission and combining for MIMO systems," in Proc. IEEE Int. Conf. Commun., vol. 5, May 2003, pp. 3205-3209.

[6] H. Samra, and Z. Ding, "New MIMO ARQ protocols and joint detection via sphere decoding," IEEE Trans. Sig. Proc. vol. 54, no. 2, pp. 473-482, Feb. 2006.

[7] A. Nakajima, D. Garg, and F. Adachi, "Throughput of turbo coded hybrid ARQ using single-carrier MIMO multiplexing," 61st IEEE veh. technol. conf. VTC'05 Spring, Stockholm, Sweden, 2005.

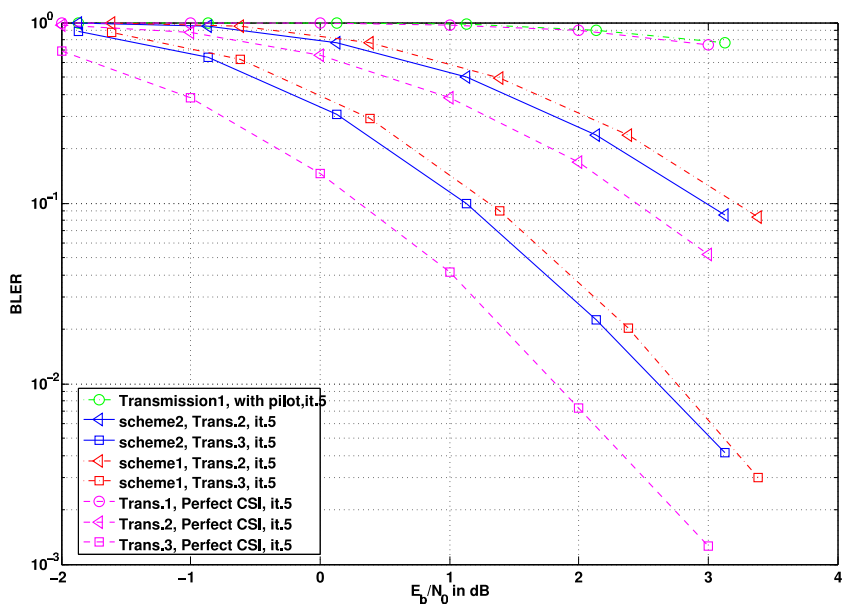

Fig. 8. BLER performance of the proposed receivers for an STBICM with $N_{T}=N_{R}=2,2 \mathrm{EQ}$ channel profile, and iterative channel estimation

[8] A. Nakajima, and F. Adachi, "Iterative joint PIC and 2D MMSE-FDE for turbo-coded HARQ with SC-MIMO multiplexing," 63rd IEEE veh. technol. conf. VTC'06 Spring, pp. 2503-2507, Melbourne, Australia, May. 2006.

[9] S. L. Ariyavisitakul, "Turbo space-time processing to improve wireless channel capacity," IEEE Commun. Conf., vol. 3, pp. 1238-1242, Jun. 2000.

[10] A. M. Tonello, "MIMO MAP equalization and turbo decoding in interleaved space time coded systems", IEEE Trans. Commun., vol. 51, no. 2, pp. 155-160, Feb. 2003.

[11] R. Visoz, and A. O. Berthet, "Iterative decoding and channel estimation for space-time BICM over MIMO block fading multipath AWGN channel", IEEE Trans. Commun., vol. 51, no. 8, pp. 1358-1367, Aug. 2003.

[12] X. Wautelet, A. Dejonghe, and L. Vandendorpe, "MMSE-based fractional turbo receiver for space-time BICM over frequency selective MIMO fading channels," IEEE Trans. Sig. Proc., vol. SIG-52, pp. 18041809, Jun. 2004

[13] R. Visoz, A. O. Berthet, and S.Chtourou, "A new class of iterative equalizers for space-time BICM over MIMO block fading multipath AWGN channel," IEEE Trans. Commun., vol. 53, no. 12, pp. 20762091, Dec. 2005.

[14] T. Ait-Idir, S. Saoudi, and N. Naja, "Space-time turbo equalization with successive interference cancellation for frequency selective MIMO channels," IEEE Trans. Veh. Technol. In Press, May 2008.

[15] M. Yee, M. Sandell, and Y. Sun, "Comparison study of single-carrier and multi-carrier modulation using iterative based receiver for MIMO systems," IEEE Veh. Technol. Conf. (VTC), vol. 3, pp. 1275-1279, Milan, Italy, May 2004.

[16] R. Visoz, A. O. Berthet, and S. Chtourou, "Frequency-domain block turbo-equalization for single-carrier transmission over MIMO broadband wireless channel," IEEE Trans. Commun., vol. 54, no. 12, pp. 2144-2149, Dec. 2006.

[17] H. Samra, and Z. Ding, "A hybrid ARQ protocol using integrated channel equalization," IEEE Trans. Commun., vol. 53, no. 12, pp. 19962001, Dec. 2005.

[18] D. N. Doan, and K. R. Narayanan, "Iterative packet combining schemes for intersymbol interference channels," IEEE Trans. Commun., vol. 50, no. 4, pp. 560-570, Apr. 2002.

[19] K. Narayanan, and G. Stuber, "A novel ARQ technique using the turbo coding principle," IEEE Commun. Lett., vol. 1, no. 3, pp. 49-51, Mar. 1997.

[20] L. R. Bahl, J. Cocke, F. Jelinek, and J. Raviv, "Optimal decoding of linear codes for minimizing symbol error rate," IEEE Trans. Inform. Theory, vol. IT-20, pp. 284-287, Mar. 1974.

[21] T. Ait-Idir, and S. Saoudi, "Low-Complexity MMSE Turbo Equalization with Successive Interference Cancellation for MIMO-ISI Channels," 16th IEEE Personal Mobile Radio Conf., Berlin, Germany, Sep. 2005. 